\title{
Exploring the Pharmacological Mechanism of the modified Chinese medicine formulas "Shenfu- Linguizhugan decoction" treating Dilated cardiomyopathy Based on Network Pharmacology
}

\section{Wuxia Quan}

Qinyang people's Hospital

Yandong Miao ( $\nabla$ miaoyd19@lzu.edu.cn )

Lanzhou University https://orcid.org/0000-0002-1429-8915

\section{Research}

Keywords: Network Pharmacology, Traditional Chinese Medicine, Shenfu Linguizhugan decoction, Dilated cardiomyopathy, Pharmacological evaluation

Posted Date: March 30th, 2020

DOI: https://doi.org/10.21203/rs.3.rs-19020/v1

License: (c) (1) This work is licensed under a Creative Commons Attribution 4.0 International License. Read Full License 


\section{Abstract}

Background: Dilated cardiomyopathy (DCM) is a non-ischaemic cardiac muscle disease with structural and functional myocardial aberration can lead to extensive morbidity and mortality due to complications in particular heart failure and arrhythmia. Two classic Chinese medicine formulas, Shenfu decoction and Linguizhugan decoction, were both shown to exert therapeutic effects on heart disease. Thus, modified Shenfu and Linguizhugan decoction (SFLGZGD) is recommended for treatment DCM. However, its chemical and pharmacological characteristics remain to be elucidated. In the current study, a network pharmacology approach was applied to characterize the action mechanism and target genes of SFLGZGD on DCM.

Methods: The gene expression of DCM was obtained from the Gene Expression Omnibus (GEO). All compounds were obtained from the correlative databases, and active mixture were selected according to their oral bioavailability (OB) and drug-likeness (DL) index. The potential targets of SFLGZGD were obtained from the traditional Chinese medicine systems pharmacology (TCMSP) database. The compound-target and target-pathway networks were constructed. The protein-protein interactive (PPI) network generated by R software was visualized by Cytoscape, and the topology scores, functional regions, and gene annotations were analyzed using plugins of Bisogenet and CytoNCA. The potential pathways related to target genes were determined by gene ontology (GO) and Kyoto Encyclopedia of Genes and Genomes (KEGG) pathway enrichment analyses.

Results: A total of 963 differentially expressed genes (DEGs), including 538 upregulated genes and 425 downregulated, were obtained from GSE19303. A total of 636 ingredients in SFLGZGD were obtained, among which, 93 were chosen as bioactive components. The compound-target network included 10 bioactive components and 18 potential targets and a total of 1939 genes obtained in the PPI network, among them, a total of 16 genes were screened out. Moreover, 129 terms on the $\mathrm{GO}$ analysis and six pathways obtained. Among these potential targets, EGFR, CDKN1A, MMP1, COL1A1, COL3A1, MMP3, ICAM1, and HSPB1 were identified as relatively high-degree targets.

Conclusions: The network pharmacology-based approach in the current study has shown promising potential in identifying major therapeutic targets from TCM formulations. Besides, our study suggested that network pharmacology prediction may provide a useful tool for describing the molecular mechanism of SFLGZGD on DCM.

\section{Background}

Dilated cardiomyopathy (DCM) is a non-ischaemic cardiac muscle disease with structural and functional myocardial aberration by the presence of left ventricular ectasia and contractile dysfunction [1-3]. The WHO defines DCM as serious cardiac disarray that can lead to extensive morbidity and mortality just that complications in particular heart failure and arrhythmia [4]. Mutations in genes that encode sarcomere, cytoskeleton, and nuclear membrane proteins accounted for 35 percent of cases [1,3]. Because of DCM 
has a large number of genes and alleles, genetic diagnosis can help predict prognosis, especially the risk of arrhythmia in some subtypes [5]. Traditional Chinese Medicine (TCM) is one of the most valuable treasures of Chinese culture, and it has been used clinically in East and Southeast Asia for over 2,000 years. As one of the most popular models of complementary and alternative medicine in China, TCM is gradually accepted by non-Chinese people due to its apparent efficacy, abundant resources, and low toxicity, and is increasingly used in western countries $[6,7]$. In the clinical treatment of DCM, there are many proprietary Chinese medicines and TCM injections alone or as an adjuvant to conventional chemotherapy [8-11].

Shen fu and Lin Gui Zhu Gan decoction both are commonly used TCM formulas. Shen Fu decoction consists of only two herbs, Ginsen Radix Et Rhizoma Rubra (GRERR, Hong-shen in Chinese) and Aconiti Lateralis Radix Praeparata (ALRP, Fu-zi in Chinese), which was widely applied for the treatment chronic heart failure [12]. Lin Gui Zhu Gan decoction consists of four TCMs, Poria Cocos (PC, Fu-lin in Chinese), Cinnamomi Ramulus (CR, Gui-zhi in Chinese), Atractylodes Macrocephala Koidz (AMK, Bai-zhu in Chinese), and Licorice (Gan-cao in Chinese), which also can be used to treat chronic heart failure [13]. Hedysarum Multijugum Maxim (HMM, Huang-qi in Chinese), as a plant of the genus astragalus in the leguminous family, which was widely applied for the treatment of various diseases, also including DCM $[14,15]$. Based on the research, therefore, modified Shen fu decoction combination Lin gui zhu gan decoction (SFLGZGD) is recommended to be applied in the TCM treatment scheme of cardiomyopathy in China. However, the chemical and pharmacological foundations of SFLGZGD in treating heart disease, especially DCM, was not comprehensively assessed by suitable approaches.

TCM is a complicated system with multiple targets and complex interactions among its components [6]. However, unlike chemical drugs found for specific proteins, the understanding of the molecular basis of TCM is still limited, and the study of modern TCM theories is lagging behind, which has slowed the development of novel TCM drugs [16]. With the rapid development in big data, bioinformatics, systems biology, and multi-pharmacology, network-based drug discovery is considered to be a promising low-cost approach to drug development. Based on the system biology method, the concept of network pharmacology of traditional Chinese medicine was first proposed in 2014 [17]. With drug discovery facing a significant bottleneck, drug research and development has gradually changed from the current "single target, single drug" model to the "network target, multi-component therapy" model [18]. It is helpful to evaluate the rationality and compatibility of TCM by providing a specific composite target and target-path network. In addition, network pharmacology methods have been used to study "complex protein/genedisease" pathways that can describe the complexity between biological systems, drugs, and diseases from a network perspective, sharing a holistic philosophy similar to that of TCM. The application of systematic biological methods to determine the pharmacological action, mechanism, and safety of TCM are of great value to the research and development of modern TCM. It has been widely applied in the research on the mechanism of TCM treatment of complex diseases, such as ischemic stroke, cancer, chronic atrophic gastritis, type 2 diabetes, and cardiovascular disease [6, 19-22]. 
In the present study, bioinformatics analysis was used to study the pharmacological network of SFLGZGD on DCM, and to predict its active components and potential target genes. In addition, we performed GO and KEGG pathway analyses to explore the functions and pathways involved. The detailed flow chart of the current study was shown in Fig. 1.

\section{Methods}

\section{Differential gene expression analysis}

The expression of gene extracted from the Gene expression dataset (GSE19303) [23], containing 73 DCM patients and 8 normal controls, which was based on [HG-U133_Plus_2] Affymetrix Human Genome U133 Plus 2.0 Array, was obtained from the NCBI Gene Expression Omnibus (GEO) (http://www.ncbi.nlm.nih.gov/geo/) and normalized by R software (version 3.6.2) and Strawberry Perl (5.30.1.1) The differential expressed genes (DEGs) between the DCM patients and normal controls were extract based on the Wilcox Test and the data annotation by the R/Bioconductor Limma package [24], DEGs with fold change value $>1, P<0.05$ were set as the cutoffs. Bidirectional hierarchical clustering analyzed and drew heat map by R-package "pheatmap"(https://cran.rproject.org/web/packages/pheatmap/).

\section{Network Pharmacology-Based Analysis of TCM}

\section{Identification of Candidate Components in the modified SFLGZGD}

All components of the modified two Chinese medicinal decoctions, including GRERR, ALRP, PC, CR, AMK, HMM, Alisma Orientale (Sam.) Juz. (AO, Ze-xie in Chinese), and Plantaginis Semen (PS, Che-qianzi), were obtained from the Traditional Chinese Medicine Systems Pharmacology Database and Analysis Platform, TCMSP) (http://tcmspw.com/) [25].

\section{Selecting procedure for Bioactive constituent in SFLGZGD}

Oral TCM decoction must overcome the obstacles caused by the process of absorption, distribution, metabolism and excretion (ADME) to be active. During the ADME processes, oral bioavailability (OB) is one of the most considerable pharmacokinetic parameters [26]. High OB is usually a crucial barometer to determine the drug-likeness (DL) index of active ingredients. The ingredients with $\mathrm{OB} \geq 30 \%$ were regarded to have high $\mathrm{OB}$. As a qualitative concept for drug design, the DL index can be used to evaluate the pharmacist of molecules, which has great significance for the rapid screening of active substances. The average $D L$ index is 0.18 in the Drug Bank database. Substances with a DL index $\geq 0.18$ are considered to have high pharmacy ability. Consequently, the compounds in SFLGZGD with OB $\geq 30 \%$ and DL index $\geq 0.18$ were selected as active substances in this study. The protein targets of the active ingredients in SFLGZGD were obtained from the TCMSP database.

\section{Construction regulatory network of TCM compounds and PPI network}


To further explore the molecular mechanism of SFLGZGD on DCM, the compound-target and targetpathway regulatory networks were generated using Cytoscape software (version 3.7.2, http://www.cytoscape.org), which is a JAVA-based network analysis and visualization tool [27]. We obtained the gene list, nodes, compound-target, and target- pathway interactions using the Perl Script. In these regulatory networks, the compounds, proteins, or pathways were performed as nodes, whereas the compound-target or target- pathway interactions were expressed as edges.

Protein-protein interactive (PPI) network is the basic frame for proteins to determine their function in the system biology. In the current work, the PPI network generated by Bisogenet plugin (version 3.0.0) [28], which integrated several databases, including Database of Interacting Protein (DIP), Biological General Repository for Interaction Datasets (BioGRID), Human Protein Reference Database (HPRD), molecular interaction Database (MINT), and biomolecular interaction network database (BIND). The method is input nodes and their neighbors. The topology scores of these nodes were measured using CytoNCA plugin (version 2.1.6) [29], which integrated calculation, evaluation, and visualization analysis for multiple centrality, including degree centrality (DC) and betweenness centrality (BC), "Without weight" was set as the parameter. The default parameters of $D C$ min value are 61 , and the $B C$ min value are 600 as cutoffs.

\section{Gene ontology and pathway enrichment analysis}

Functional enrichment analysis of compound-target or target- pathway was based on the R/Bioconductor "clusterProfiler," "org.Hs.eg.db," "enrichplot," and "ggplot2" package, which identified Gene Ontology (GO) categories in the Biological Processes (BP), Cellular Components (CC), or Molecular Functions (MF) and Kyoto Encyclopedia of Genes and Genomes (KEGG) pathway analysis to reveal bio-information of the identified genes, both up- and down- regulated. Count $\geq 2$ and FDR 0.05 were set as a significance threshold to determine enrichment terms. Moreover, we constructed a relational network of KEGG by Perl Script and Cytoscape software.

\section{Results}

A total of 963 DEGs, including 538 upregulated genes and 425 downregulated, were obtained from DCMexpression microarray dataset GSE19303, which was statistical significance between normal controls and DCM patients. The TOP 20 genes, both in the upregulated and downregulated groups, are shown in the heatmap and all the DEGs through volcano plot filtering (Additional file 1: Fig. 1). A detailed list of the 936 genes and the corresponding statistics is shown in Additional file 1.

\section{Network Pharmacology-Based Analysis of TCM}

\section{Identification of Bioactive Components in the SFLGZGD}

A total of 636 ingredients in SFLGZGD were obtained from the TCMSP database. Among the 636 components in SFLGZGD, 324 (50.9\%) met the requirement of OB $\geq 30 \%$ and 93 (14.6\%) met the 
requirements of $O B \geq 30 \%$, and $D L$ index $\geq 0.18$. The whole components list manifest in Table 1 , and the detailed list of the 93 candidate Bioactive Components as shown in Additional file 2.

\section{Compound-Target Network and PPI network}

\section{Herb-Compound-Compound Target Network}

Exploring the molecular basis of TCM is very crucial for the modernization of TCM, and understanding the targets of TCM is momentous. In the present study, the compound-target network of SFLGZGD on DCM was constructed (Fig.2), which was composed of 28 nodes (10 for bioactive components and 18 for potential targets). These potential targets, including ACHE, MMP3, EGFR, CDKN1A, MMP1, ICAM1, PTGER3, HSPB1, MGAM, COL1A1, ABCG2, PSMD3, COL3A1, CLDN4, CTSD, IGFBP3, MTTP, and ND6, associated with the 10 bioactive components. Except for the MOL000098 derived from PS and HMM, and the rest of bioactive ingredients comes from HMM, CR, ALRP, AMK, and PS, respectively, and the detailed information was shown in Table 2, which signify that these five TCMs and these targets in the network play a significant effect in the process of SFLGZGD treating DCM.

\section{PPI Network Analysis}

The PPI network was constructed by the Bisogenet plugin. A total of 1939 genes obtained, the degree ranged from 1 to 933 (Additional file: Fig. 2). The topologically essential genes screened by CytoNCA, a total of 249 genes selected by the parameter "Without weight" and DC min value greater than 81, then, we generated the sub-network based on the previously filtered data, which was shown in the Fig.3A. Besides, we constructed the network by the parameter "Without weight" and BC min value greater than 600 based on the sub-network of previous 249 genes, a total of 16 genes were screened out (Fig.3B). Finally, we also generated the sub-network, these genes in the network may account for the significantly essential therapeutic effects of SFLGZGD on DMC, especially the high-degree protein targets, such as EGFR (degree=933), NTRK1 (degree=674), and HSPB1 (degree=415). The detailed PPI information was shown in Additional file 3 .

\section{Gene ontology enrichment and KEGG pathway analysis}

To identify the biological characteristics of presumptive targets of SFLGZGD on DCM in detail, the GO and KEGG pathway enrichment analyses of involved targets were conducted using several R-packages by R software. We obtained 129 terms on the GO analysis, including 94 BP, 25 CC, and 10 MF, respectively, Count $\geq 2$ and FDR囚0.05 were as cutoffs (Fig. 4). The detailed GO information was manifested in Additional file 4. The top 20 significantly enriched terms in BP, CC, and MF categories were manifested in Fig. 5. There are 6 genes fall into the category of extracellular structure organization (FDR $=1.15 \mathrm{E}-03$ ), 5 genes fall into the category of response to toxic substance (FDR $=7.71 \mathrm{E}-03)$, multicellular organismal homeostasis (FDR $=6.39 \mathrm{E}-03)$, response to oxidative stress (FDR $=5.53 \mathrm{E}-03)$, response to steroid hormone (FDR $=3.75 \mathrm{E}-03)$, and extracellular matrix organization (FDR $=2.65 \mathrm{E}-03)$, respectively, which were the top 6 terms of BP having the largest number of genes enriched (Fig. 5A). The top 3 terms of CC 
annotation showed that the gene products mainly enriched in the extracellular matrix (7 genes), collagencontaining extracellular matrix (5 genes) and endoplasmic reticulum lumen (4 genes) (Fig. 5B). As to MF annotation, integrin binding, growth factor binding, and serine hydrolase activity were the most enriched term with 3 genes hits, respectively (Fig. 5C).

The KEGG enrichment analysis revealed that 6 pathways were significantly associated with targets of SFLGZGD on DCM as shown in Fig. 6 and Table 3. Among these potential pathways, "Relaxin signaling pathway" was considered the most significant one with the highest degree value (Fig. 6A). Among these potential targets, EGFR, CDKN1A, MMP1, COL1A1, COL3A1, MMP3, ICAM1, and HSPB1 were identified as relatively high-degree targets, which played a crucial role in the development of DCM and were considered as the key markers of SFLGZGD treatment on DCM (Fig. 6B). From the incorporated drug target prediction, GO, and pathway enrichment as well as network analyses, we speculated that the effects of SFLGZGD on DCM might be associated with the roles of its key targets including EGFR, MMP1, COL1A1, COL3A1, CDKN1A, MMP3, ICAM1, and HSPB1 in regulating myocardial cell function.

\section{Discussion}

DCM is a non-ischaemic cardiac muscle disease with structural and functional myocardial malformations. Gene mutation can induce DCM, including genes encoding structural elements of the sarcomere and desmosome [2]. In the present study, we obtained the DCM gene data from the GSE19303 dataset using the bioinformatics analysis method and identified 538 genes that might involve in this disease. TCM uses therapeutic herbs to treat diseases based on a patient's syndrome and to recover from a balance of life and body functions [30] and may manifest extensive pharmacological activities with multiple targets and pathways [31], which may benefit the therapy of DCM. However, this characteristic may bring difficulty to lucubrate the internal mechanism of TCM. The Network pharmacology approach, which based on the rapid progress in bioinformatics, systems biology, and polypharmacology, may offer a promising approach for the mechanistic study of intricated TCM. In the traditional Chinese medicine system, compounds lacking appropriate pharmacokinetic properties cannot reach the target organ to convey biological activities. In the present study, the SFLGZGD mixture with OB $\geq 30 \%$ and DL index $\geq 0.18$ were considered pharmacokinetically active, which may be likelihood absorbed and distributed in the human body. In the ingredients-target network, ingredients with high-degree may account for the major therapeutic effects of SFLGZGD on DCM.

In the present study, the compound-target network of SFLGZGD on DCM was constructed, which was composed of 10 candidate bioactive components and 18 potential targets. Among the network, only MOL000098 derived from two herbs, Plantaginis Semen and Hedysarum Multijugum Maxim, which means quercetin plays a crucial role in the treatment of DCM. MOL000098 (quercetin), a polyphenolic flavonoid with potential chemopreventive activity, has been found to be effective in alleviating experimental autoimmune myocarditis and have therapeutic benefit in cardiovascular diseases[32, 33], in addition to, quercetin protecting the heart of the rats with post myocarditis DCM maybe involves the modulation of mitogen-activated protein kinases (MAPK) signal cascade[34]. Five of the 10 candidate 
bioactive components derived from Hedysarum Multijugum Maxim, which means it plays a crucial role in the treatment of DCM, especially, the bioactive components, MOL000422, MOL000380, MOL000371, MOL000392, and MOL000354. MOL000422 (kaempferol) can moderate cardiac injuries by inhibiting inflammatory responses and oxidative stress and mechanically linked to inhibition of nuclear factor-KB (NFkB) and nuclear factor-erythroid 2 p45-related factor-2 activation [35]. Moreover, during the animal experiment, MOL000392 (formononetin) can inhibit atherogenic monocyte adhesion and inflammation by regulating the interaction between KLF4 and SRA in ApoE deficient mice[36]. Besides, formononetin protected cardiomyocytes from oxygen-glucose deprivation/reoxygenation injury via inhibiting reactive oxygen species formation and promoting glycogen synthase kinase $3 \beta$ phosphorylation [37]. Previous reports have shown that MOL000354 (isorhamnetin) can exhibit a positive effect on injuring cardiomyocytes by upregulation of SIRT1 and Nrf2/HO-1-mediated antioxidant signaling pathway, protective targeting cardiac hypertrophy by blocking the phosphatidylinositol 3-kinase-AKT signaling pathway [38, 39]. Furthermore, MOL004576 (taxifolin), a plant flavonoid separated from yew, can alleviate cardiac hypertrophy by activating the IL-6/JAK/STAT3 signal pathway, triggering glycometabolism disorder and mitochondrial dysfunction in vitro [40], and also remarkably improve the cardiac function, regulated oxidative stress and attenuated apoptosis pathway [41]. However, there was no literature relating to MOL000380, MOL000371, MOL002392, MOL000049, and MOL001735 with DCM. All these previous literatures, combined with our study, supported the conclusion of network prediction and illustrated a successful practice of network pharmacology approach in the identification mechanism of effect in TCM.

In the PPI network, the top 16 high ranking genes were identified via CytoNCA based on the importance connectivity of them in the whole network. Most of them have established well known association with the etiology and mechanism of DCM, especially NTRK1, EGFR, TP53, CUL3, UBC, and FN1, these genes ranked in the top six, both in the $\mathrm{DC}$ and $\mathrm{BC}$ groups. ErbB2 signaling in cardiomyocytes is essential for the prevention of dilated cardiomyopathy [42]and ErbB2 signaling changes maybe cause cardiomyopathy [43]. TP53, encodes a tumor suppressor protein, mutations in this gene are associated with a variety of human cancers. However, it also played a role in the development of DCM. TP53 signaling pathway was activated in the heart tissue of DCM patients with ventricular tachycardia [44] and LMNA (Lamin A/C) Mutations [45]. FN1, as a member of extracellular matrix-encoding genes, Significant upregulation of structural was evident from the early, pre-phenotype stage of severe Hypertrophic cardiomyopathy (HCM) and heart failure [46]. Further, more detailed mechanisms of these genes in the DCM deserved to investigate in the future study.

Finally, the GO and KEGG pathway enrichment analyses of involved targets were conducted using several R-packages by R software to identify the biological characteristics of presumptive targets of SFLGZGD on DCM in detail. 129 enriched GO terms revealed fundamental pathophysiological alterations of DCM. These target genes mainly rich in the terms of extracellular matrix and its related, which demonstrated that SFLGZGD treats DCM patients may be via inhibit cardiomyocyte fibrosis and cardiomyocyte hypertrophy. Adverse reconstructing of the extracellular matrix (ECM) is a significant feature of heart failure. ECM reconstructing is extensive in several heart diseases and hinders cardiac filling, often leading 
to heart failure [47-49]. Response to oxidative stress, steroid hormone are also leading causes of adverse remodel reconstructing during DCM development [50, 51]. Furthermore, 8 genes (EGFR, CDKN1A, MMP1, COL1A1, COL3A1, MMP3, ICAM1, and HSPB1) identified by KEGG analysis were significantly enriched pathway terms, including Relaxin signaling pathway, bladder cancer, rheumatoid arthritis, prostate cancer, AGE-RAGE signaling pathway in diabetic complications, and amoebiasis. EGFR is a transmembrane glucoprotein that is a member of the protein kinase superfamily, and a receptor of the epidermal growth factor family, binding of the protein to a ligand induces receptor dimerization and leads to cell proliferation. Modulating EGFR transactivation and signal is a fundamental mechanism during DCM development $[52,53]$. Specifically, ErbB2 signaling in cardiomyocytes is, therefore, essential for the prevention of dilated cardiomyopathy. CDKN1A encodes a potent cyclin-dependent kinase inhibitor to inhibit the activity of cyclin-cyclin-dependent kinase 2 or -cyclin-dependent kinase4 complexes, as a regulator of cell cycle progression at G1. Previous study demonstrated that CDKN1A could regulate cardiomyocyte proliferation during heart development [54]. MMP1 encodes a member of the proteinase M10 family of matrix metalloproteinases (MMPs). Proteins in this family are involved in the breakdown of extracellular matrix in normal physiological processes, such as embryonic development, reproduction, and tissue remodeling, as well as in disease processes, such as arthritis and metastasis. MMPs play a key role in myocardial remodel and heart failure $[55,56]$. COL1A1 encodes the pro-alpha 1 chains of type I collagen whose triple helix comprises two alpha1 chains and one alpha2 chain. Collagen-derived peptides have been conventionally used as markers of cardiac fibrosis [57], and increased expression and labeling of collagen in DCM samples indicate fibrosis may contribute to t-tubule remodel in the human heart failure [58]. In addition, there are studies shown that Collagen I and MMP-1 immunoexpression in DCM [59] and MMP3 has a correlation with DCM [60]. HSPB1 encodes a member of the small heat shock

protein family of proteins. HSPB1 serves as a negative regulator of NFKB-mediated leucocyte recruitment, and the subsequent inflammation in cardiomyocytes [61] and HSPB1 in the failing heart following myocardial infarction were decreased [62]. In general, these genes are hub genes of SFLGZGD treating on DCM and further detailed pharmacological mechanisms by which SFLGZGD treating DCM will be investigated in our future study.

\section{Conclusion}

In conclusion, the pharmacological mechanism by which SFLGZGD treating DCM was investigated with the combination of TCM-network pharmacology prediction. We demonstrated that SFLGZGD might inhibit cardiomyocyte fibrosis and cardiomyocyte hypertrophy to treat DCM patients, which mainly via the regulation of the extracellular matrix, extracellular structure organization, and Relaxin signaling pathway. Our study further suggested that TCM-network pharmacology prediction may offer a useful tool to characterize the action mechanism of TCM in detail. We underline a central role of some hub genes such as EGFR, CDKN1A, and MMP1 in the process of SFLGZGD treating on DCM, which may have diagnostic and therapeutic value in the future. The potential therapeutic effects of SFLGZGD on DCM may benefit from further studies on clinical trials of DCC patients with SFLGZGD treatment. 


\section{Abbreviations}

DCM: Dilated cardiomyopathy; SFLGZGD: Shenfu-Linguizhugan decoction; GEO: Gene Expression Omnibus; OB: Oral bioavailability; DL: Drug-likeness; TCMSP: Traditional Chinese medicine systems pharmacology; GO: gene ontology; KEGG: Kyoto Encyclopedia of Genes and Genomes; DEGs:

Differentially expressed genes; GRERR: Ginsen Radix Et Rhizoma Rubra; ALRP: Aconiti Lateralis Radix Praeparata; PC:Poria Cocos; CR: Cinnamomi Ramulus; AMK: Atractylodes Macrocephala Koidz; HMM: Hedysarum Multijugum Maxim; AO: Alisma Orientale; PS: Plantaginis Semen; ADME:absorption, distribution, metabolism and excretion; PPI: Protein-protein interactive ; Database of Interacting Protein (DIP), Biological General Repository for Interaction Datasets (BioGRID), Human Protein Reference Database (HPRD), molecular interaction Database (MINT), and biomolecular interaction network database (BIND). DC: degree centrality; BC: betweenness centrality; GO: Gene Ontology; BP: Biological Processes; CC: Cellular Components; MF:Molecular Functions; KEGG: Kyoto Encyclopedia of Genes and Genomes; mitogen-activated protein kinases (MAPK); NFKB: nuclear factor-kB; ECM: extracellular matrix.

\section{Declarations}

\section{Acknowledgements}

We thank the GEO and TCMSP databases for the availability of the data.

\section{Authors' contributions}

WXQ and YDM designed and conducted the study with equal contribution. WXQ downloaded, and integrated data, then drafted the article. YDM analyzed data and revise the article. All authors read and approval of the final manuscript.

\section{Funding}

This research did not receive any specific grant from funding agencies in the public, commercial, or notfor-profit sectors.

\section{Availability of data and materials}

The authors certify that all the original data in this research could be obtained from public database. The datasets used and/or analysed during the current study are available from the corresponding author on reasonable request.

\section{Ethics approval and consent to participate}

Not applicable.

\section{Consent for publication}


Not applicable.

Competing interests

The authors declare that they have no competing interests.

\section{References}

1. Weintraub RG, Semsarian C, Macdonald P: Dilated cardiomyopathy. Lancet (London, England) 2017, 390(10092):400-414.

2. Schultheiss HP, Fairweather D, Caforio ALP, Escher F, Hershberger RE, Lipshultz SE, Liu PP, Matsumori A, Mazzanti A, McMurray J et al: Dilated cardiomyopathy. Nature reviews Disease primers 2019, 5(1):32.

3. Hershberger RE, Morales A, Siegfried JD: Clinical and genetic issues in dilated cardiomyopathy: a review for genetics professionals. Genetics in medicine : official journal of the American College of Medical Genetics 2010, 12(11):655-667.

4. Richardson P, McKenna W, Bristow M, Maisch B, Mautner B, O'Connell J, Olsen E, Thiene G, Goodwin $\mathrm{J}$, Gyarfas I et al: Report of the 1995 World Health Organization/International Society and Federation of Cardiology Task Force on the Definition and Classification of cardiomyopathies. Circulation 1996, 93(5):841-842.

5. McNally EM, Mestroni L: Dilated Cardiomyopathy: Genetic Determinants and Mechanisms. Circulation research 2017, 121(7):731-748.

6. Guo W, Huang J, Wang N, Tan HY, Cheung F, Chen F, Feng Y: Integrating Network Pharmacology and Pharmacological Evaluation for Deciphering the Action Mechanism of Herbal Formula Zuojin Pill in Suppressing Hepatocellular Carcinoma. Front Pharmaco/ 2019, 10:1185.

7. Cheung F: TCM: Made in China. Nature 2011, 480(7378):S82-83.

8. Shen FF, Jiang TH, Jiang JQ, Lou Y, Hou XM: Traditional chinese medicine tongxinluo improves cardiac function of rats with dilated cardiomyopathy. Evidence-based complementary and alternative medicine : eCAM 2014, 2014:323870.

9. Sun R, Wang J, Zheng Y, Li X, Xie T, Li R, Liu M, Cao Y, Lu L, Zhang Q et al: Traditional Chinese medicine baoxin decoction improves cardiac fibrosis of rats with dilated cardiomyopathy. Experimental and therapeutic medicine 2017, 13(5):1900-1906.

10. Gao K, Song YP, Song A, Chen H, Zhao LT, Zhang HW: Therapeutic efficacy of shenmai injection as an adjuvant treatment in dilated cardiomyopathy: A protocol for systematic review. Medicine 2020, 99(8):e19158.

11. Yi F, Tan XL, Yan X, Liu HB: In silico profiling for secondary metabolites from Lepidium meyenii (maca) by the pharmacophore and ligand-shape-based joint approach. Chinese medicine 2016, $11: 42$. 
12. Wei $H$, Wu H, Yu W, Yan X, Zhang X: Shenfu decoction as adjuvant therapy for improving quality of life and hepatic dysfunction in patients with symptomatic chronic heart failure. Journal of ethnopharmacology 2015, 169:347-355.

13. zhang $b$, li $x$ : Discussion on the treatment of chronic heart failure with linggui jugan decoction "kaixuanfu - qi sanjiao". Chinese journal of traditional Chinese medicine 2019, 34(03):1006-1008.

14. Fu J, Wang Z, Huang L, Zheng S, Wang D, Chen S, Zhang H, Yang S: Review of the botanical characteristics, phytochemistry, and pharmacology of Astragalus membranaceus (Huangqi). Phytotherapy research : PTR 2014, 28(9):1275-1283.

15. Zhou X, Xin Q, Wang Y, Zhao Y, Chai H, Huang X, Tao X, Zhao M: Total Flavonoids of Astragalus Plays a Cardioprotective Role in Viral Myocarditis. Acta Cardiologica Sinica 2016, 32(1):81-88.

16. Zhang R, Zhu X, Bai H, Ning K: Network Pharmacology Databases for Traditional Chinese Medicine: Review and Assessment. Front Pharmacol 2019, 10:123.

17. Li S, Fan TP, Jia W, Lu A, Zhang W: Network pharmacology in traditional chinese medicine. Evidencebased complementary and alternative medicine : eCAM 2014, 2014:138460.

18. Luo M, Jiao J, Wang R: Screening drug target combinations in disease-related molecular networks. BMC bioinformatics 2019, 20(Suppl 7):198.

19. Zhao J, Lv C, Wu Q, Zeng H, Guo X, Yang J, Tian S, Zhang W: Computational systems pharmacology reveals an antiplatelet and neuroprotective mechanism of Deng-Zhan-Xi-Xin injection in the treatment of ischemic stroke. Pharmacological research 2019, 147:104365.

20. Tian G, Wu C, Li J, Liang B, Zhang F, Fan X, Li Z, Wang Y, Li Z, Liu D et al: Network pharmacology based investigation into the effect and mechanism of Modified Sijunzi Decoction against the subtypes of chronic atrophic gastritis. Pharmacological research 2019, 144:158-166.

21. Gao K, Yang R, Zhang J, Wang Z, Jia C, Zhang F, Li S, Wang J, Murtaza G, Xie H et al: Effects of Qijian mixture on type 2 diabetes assessed by metabonomics, gut microbiota and network pharmacology. Pharmacological research 2018, 130:93-109.

22. Tao W, Xu X, Wang X, Li B, Wang Y, Li Y, Yang L: Network pharmacology-based prediction of the active ingredients and potential targets of Chinese herbal Radix Curcumae formula for application to cardiovascular disease. Journal of ethnopharmacology 2013, 145(1):1-10.

23. Ameling S, Bhardwaj G, Hammer E, Beug D, Steil L, Reinke Y, Weitmann K, Grube M, Trimpert C, Klingel $\mathrm{K}$ et al: Changes of myocardial gene expression and protein composition in patients with dilated cardiomyopathy after immunoadsorption with subsequent immunoglobulin substitution. Basic research in cardiology 2016, 111(5):53.

24. Ritchie ME, Phipson B, Wu D, Hu Y, Law CW, Shi W, Smyth GK: limma powers differential expression analyses for RNA-sequencing and microarray studies. Nucleic acids research 2015, 43(7):e47.

25. Ru J, Li P, Wang J, Zhou W, Li B, Huang C, Li P, Guo Z, Tao W, Yang Y et al: TCMSP: a database of systems pharmacology for drug discovery from herbal medicines. Journal of cheminformatics 2014, 6:13. 
26. Xu X, Zhang W, Huang C, Li Y, Yu H, Wang Y, Duan J, Ling Y: A novel chemometric method for the prediction of human oral bioavailability. International journal of molecular sciences 2012 , 13(6):6964-6982.

27. Shannon P, Markiel A, Ozier O, Baliga NS, Wang JT, Ramage D, Amin N, Schwikowski B, Ideker T: Cytoscape: a software environment for integrated models of biomolecular interaction networks. Genome research 2003, 13(11):2498-2504.

28. Martin A, Ochagavia ME, Rabasa LC, Miranda J, Fernandez-de-Cossio J, Bringas R: BisoGenet: a new tool for gene network building, visualization and analysis. BMC bioinformatics 2010, 11:91.

29. Lv Y, He XY, Li D, Liu T, Wen GQ, Li J: Sex-specific and opposite modulatory aspects revealed by PPI network and pathway analysis of ischemic stroke in humans. PloS one 2020, 15(1):e0227481.

30. Qiu J: Traditional medicine: a culture in the balance. Nature 2007, 448(7150):126-128.

31. Zhou X, Menche J, Barabasi AL, Sharma A: Human symptoms-disease network. Nature communications 2014, 5:4212.

32. Javadi B, Sahebkar A: Natural products with anti-inflammatory and immunomodulatory activities against autoimmune myocarditis. Pharmacological research 2017, 124:34-42.

33. Perez-Vizcaino F, Duarte J, Andriantsitohaina R: Endothelial function and cardiovascular disease: effects of quercetin and wine polyphenols. Free radical research 2006, 40(10):1054-1065.

34. Arumugam S, Thandavarayan RA, Arozal W, Sari FR, Giridharan VV, Soetikno V, Palaniyandi SS, Harima M, Suzuki K, Nagata M et al: Quercetin offers cardioprotection against progression of experimental autoimmune myocarditis by suppression of oxidative and endoplasmic reticulum stress via endothelin-1/MAPK signalling. Free radical research 2012, 46(2):154-163.

35. Chen X, Qian J, Wang L, Li J, Zhao Y, Han J, Khan Z, Chen X, Wang J, Liang G: Kaempferol attenuates hyperglycemia-induced cardiac injuries by inhibiting inflammatory responses and oxidative stress. Endocrine 2018, 60(1):83-94.

36. Ma C, Xia R, Yang S, Liu L, Zhang J, Feng K, Shang Y, Qu J, Li L, Chen N et al: Formononetin attenuates atherosclerosis via regulating interaction between KLF4 and SRA in apoE(-/-) mice. Theranostics 2020, 10(3):1090-1106.

37. Cheng Y, Xia Z, Han Y, Rong J: Plant Natural Product Formononetin Protects Rat Cardiomyocyte H9c2 Cells against Oxygen Glucose Deprivation and Reoxygenation via Inhibiting ROS Formation and Promoting GSK-3beta Phosphorylation. Oxidative medicine and cellular longevity 2016, 2016:2060874.

38. Zhao TT, Yang TL, Gong L, Wu P: Isorhamnetin protects against hypoxia/reoxygenation-induced injure by attenuating apoptosis and oxidative stress in H9c2 cardiomyocytes. Gene 2018, 666:92-99.

39. Gao L, Yao R, Liu Y, Wang Z, Huang Z, Du B, Zhang D, Wu L, Xiao L, Zhang Y: Isorhamnetin protects against cardiac hypertrophy through blocking PI3K-AKT pathway. Molecular and cellular biochemistry 2017, 429(1-2):167-177.

40. Cai J, Shi G, Zhang Y, Zheng Y, Yang J, Liu Q, Gong Y, Yu D, Zhang Z: Taxifolin ameliorates DEHPinduced cardiomyocyte hypertrophy via attenuating mitochondrial dysfunction and glycometabolism 
disorder in chicken. Environmental pollution (Barking, Essex : 1987) 2019, 255(Pt 1):113155.

41. Tang Z, Yang C, Zuo B, Zhang Y, Wu G, Wang Y, Wang Z: Taxifolin protects rat against myocardial ischemia/reperfusion injury by modulating the mitochondrial apoptosis pathway. PeerJ 2019, 7:e6383.

42. Crone SA, Zhao YY, Fan L, Gu Y, Minamisawa S, Liu Y, Peterson KL, Chen J, Kahn R, Condorelli G et al: ErbB2 is essential in the prevention of dilated cardiomyopathy. Nature medicine 2002, 8(5):459-465.

43. Yu L, Lee T, Lin N, Wolf MJ: Affecting Rhomboid-3 function causes a dilated heart in adult Drosophila. PLoS genetics 2010, 6(5):e1000969.

44. Haywood ME, Cocciolo A, Porter KF, Dobrinskikh E, Slavov D, Graw SL, Reece TB, Ambardekar AV, Bristow MR, Mestroni L et al: Transcriptome signature of ventricular arrhythmia in dilated cardiomyopathy reveals increased fibrosis and activated TP53. Journal of molecular and cellular cardiology 2020, 139:124-134.

45. Chen SN, Lombardi R, Karmouch J, Tsai JY, Czernuszewicz G, Taylor MRG, Mestroni L, Coarfa C, Gurha P, Marian AJ: DNA Damage Response/TP53 Pathway Is Activated and Contributes to the Pathogenesis of Dilated Cardiomyopathy Associated With LMNA (Lamin AVC) Mutations. Circulation research 2019, 124(6):856-873.

46. Tsoutsman T, Wang X, Garchow K, Riser B, Twigg S, Semsarian C: CCN2 plays a key role in extracellular matrix gene expression in severe hypertrophic cardiomyopathy and heart failure. Journal of molecular and cellular cardiology 2013, 62:164-178.

47. Christensen G, Herum KM, Lunde IG: Sweet, yet underappreciated: Proteoglycans and extracellular matrix remodeling in heart disease. Matrix biology : journal of the International Society for Matrix Biology 2019, 75-76:286-299.

48. Law ML, Cohen H, Martin AA, Angulski ABB, Metzger JM: Dysregulation of Calcium Handling in Duchenne Muscular Dystrophy-Associated Dilated Cardiomyopathy: Mechanisms and Experimental Therapeutic Strategies. Journal of clinical medicine 2020, 9(2).

49. Joanne P, Kitsara M, Boitard SE, Naemetalla H, Vanneaux V, Pernot M, Larghero J, Forest P, Chen Y, Menasche $P$ et al: Nanofibrous clinical-grade collagen scaffolds seeded with human cardiomyocytes induces cardiac remodeling in dilated cardiomyopathy. Biomaterials 2016, 80:157-168.

50. Abutaleb ARA, McNally EM, Khan SS, Anderson AS, Carr JC, Wilcox JE: Myocarditis in Duchenne Muscular Dystrophy After Changing Steroids. JAMA cardiology 2018, 3(10):1006-1010.

51. Angelini A, Gorey MA, Dumont F, Mougenot N, Chatzifrangkeskou M, Muchir A, Li Z, Mericskay M, Decaux JF: Cardioprotective effects of alpha-cardiac actin on oxidative stress in a dilated cardiomyopathy mouse model. FASEB journal : official publication of the Federation of American Societies for Experimental Biology 2020, 34(2):2987-3005.

52. Martin R, Gutierrez B, Cordova C, Roman AS, Alvarez Y, Hernandez M, Cachofeiro V, Nieto ML: Secreted Phospholipase A2-IIA Modulates Transdifferentiation of Cardiac Fibroblast through EGFR Transactivation: An Inflammation-Fibrosis Link. Cells 2020, 9(2). 
53. Reischauer S, Arnaout R, Ramadass R, Stainier DY: Actin binding GFP allows 4D in vivo imaging of myofilament dynamics in the zebrafish heart and the identification of Erbb2 signaling as a remodeling factor of myofibril architecture. Circulation research 2014, 115(10):845-856.

54. Garnatz AS, Gao Z, Broman M, Martens S, Earley JU, Svensson EC: FOG-2 mediated recruitment of the NuRD complex regulates cardiomyocyte proliferation during heart development. Developmental biology 2014, 395(1):50-61.

55. Felkin LE, Birks EJ, George R, Wong S, Khaghani A, Yacoub MH, Barton PJ: A quantitative gene expression profile of matrix metalloproteinases (MMPS) and their inhibitors (TIMPS) in the myocardium of patients with deteriorating heart failure requiring left ventricular assist device support. The Journal of heart and lung transplantation : the official publication of the International Society for Heart Transplantation 2006, 25(12):1413-1419.

56. Sakamuri SS, Takawale A, Basu R, Fedak PW, Freed D, Sergi C, Oudit GY, Kassiri Z: Differential impact of mechanical unloading on structural and nonstructural components of the extracellular matrix in advanced human heart failure. Translational research : the journal of laboratory and clinical medicine 2016, 172:30-44.

57. Nagao K, Inada T, Tamura A, Kajitani K, Shimamura K, Yukawa H, Aida K, Sowa N, Nishiga M, Horie T et al: Circulating markers of collagen types I, III, and IV in patients with dilated cardiomyopathy: relationships with myocardial collagen expression. ESC heart failure 2018, 5(6):1044-1051.

58. Crossman DJ, Shen X, Jullig M, Munro M, Hou Y, Middleditch M, Shrestha D, Li A, Lal S, Dos Remedios $C G$ et al: Increased collagen within the transverse tubules in human heart failure. Cardiovascular research 2017, 113(8):879-891.

59. Mihailovici AR, Deliu RC, Margaritescu C, Simionescu CE, Donoiu I, Istratoaie O, Tudorascu DR, Tartea EA, Gheonea DI: Collagen I and III, MMP-1 and TIMP-1 immunoexpression in dilated cardiomyopathy. Romanian journal of morphology and embryology = Revue roumaine de morphologie et embryologie 2017, 58(3):777-781.

60. Nair N, Gongora E: Correlations of GDF-15 with SST2, MMPs, and worsening functional capacity in idiopathic dilated cardiomyopathy: Can we gain new insights into the pathophysiology? Journal of circulating biomarkers 2018, 7:1849454417751735.

61. Wang Y, Liu J, Kong Q, Cheng H, Tu F, Yu P, Liu Y, Zhang X, Li C, Li Y et al: Cardiomyocyte-specific deficiency of HSPB1 worsens cardiac dysfunction by activating NFkappaB-mediated leucocyte recruitment after myocardial infarction. Cardiovascular research 2019, 115(1):154-167.

62. Marunouchi T, Inomata S, Sanbe A, Takagi N, Tanonaka K: Protective effect of geranylgeranylacetone via enhanced induction of HSPB1 and HSPB8 in mitochondria of the failing heart following myocardial infarction in rats. European journal of pharmacology 2014, 730:140-147.

\section{Tables}

Table 1 Number of ingredients in SFLGZGD with OB $\geq 30 \%$ and DL index $\geq 0.18$. 


\begin{tabular}{|c|c|c|c|c|}
\hline Herbs (chinese name) & Herbs (Latin name) & Total(n) & $\mathrm{OB} \geq 30 \%$ & $\mathrm{OB} \geq 30 \%$ and $\mathrm{DL} 0.18$ \\
\hline Hong-shen & Ginsen Radix Et Rhizoma Rubra & 74 & $23(31.1 \%)$ & $4(5.4 \%)$ \\
\hline Fu-zi & Aconiti Lateralis Radix Praeparata & 65 & $28(43.1 \%)$ & 21 (32.3\%) \\
\hline Huang-qi & Hedysarum Multijugum Maxim & 87 & $44(50.6 \%)$ & $20(32.0 \%)$ \\
\hline Fu-lin & Poria Cocos (Schw.) Wolf. & 34 & $18(52.9 \%)$ & $15(44.1 \%)$ \\
\hline Gui-zhi & Cinnamomi Ramulus & 220 & $136(61.8 \%)$ & $7(3.2 \%)$ \\
\hline Bai-zhu & Atractylodes Macrocephala Koidz & 55 & $36(65.5 \%)$ & 7 (19.4\%) \\
\hline Ze-xie & Alisma Orientale (Sam.) Juz. & 46 & $23(50.0 \%)$ & $10(21.7 \%)$ \\
\hline Che-qian-zi & Plantaginis Semen & 55 & $16(29.1 \%)$ & $9(16.4 \%)$ \\
\hline Total & & 636 & 324 (50.9\%) & $93(14.6 \%)$ \\
\hline
\end{tabular}

Table 2 Information for candidate bioactive ingredients of SFLGZGD 


\begin{tabular}{|c|c|c|c|c|c|}
\hline Number & Molecule Name & $\begin{array}{l}\text { OB } \\
(\%)\end{array}$ & DL & $\begin{array}{l}\text { Molecules } \\
\text { structure }\end{array}$ & Herb (Latin name) \\
\hline MOL004576 & taxifolin & 57.84 & 0.27 & & Cinnamomi Ramulus \\
\hline MOL000422 & kaempferol & 41.88 & 0.24 & & $\begin{array}{l}\text { Hedysarum Multijugum } \\
\text { Maxim }\end{array}$ \\
\hline MOL000380 & $\begin{array}{l}\text { (6aR,11aR)-9,10-dimethoxy-6a,11a- } \\
\text { dihydro-6H-benzofurano[3,2-c]chromen-3- } \\
\text { ol }\end{array}$ & 64.26 & 0.42 & & $\begin{array}{l}\text { Hedysarum Multijugum } \\
\text { Maxim }\end{array}$ \\
\hline MOL000371 & 3,9-di-O-methylnissolin & 53.74 & 0.48 & & $\begin{array}{l}\text { Hedysarum Multijugum } \\
\text { Maxim }\end{array}$ \\
\hline MOL000392 & formononetin & 69.67 & 0.21 & & $\begin{array}{l}\text { Hedysarum Multijugum } \\
\text { Maxim }\end{array}$ \\
\hline MOL000354 & isorhamnetin & 49.6 & 0.31 & & $\begin{array}{l}\text { Hedysarum Multijugum } \\
\text { Maxim }\end{array}$ \\
\hline MOL002392 & Deltoin & 46.69 & 0.37 & 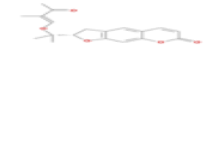 & $\begin{array}{l}\text { Aconiti Lateralis Radix } \\
\text { Praeparata }\end{array}$ \\
\hline MOL000049 & $3 \beta$ acetoxyatractylone & 54.07 & 0.22 & zorert & $\begin{array}{l}\text { Atractylodes } \\
\text { Macrocephala Koidz }\end{array}$ \\
\hline MOL001735 & Dinatin & 30.97 & 0.27 & $5 x=$ & Plantaginis Semen \\
\hline MOL000098 & quercetin & 46.43 & 0.28 & & $\begin{array}{l}\text { Plantaginis Semen and } \\
\text { Hedysarum Multijugum } \\
\text { Maxim }\end{array}$ \\
\hline
\end{tabular}

\section{Table 3 The KEGG enrichment analysis pathways.}




\begin{tabular}{|c|c|c|c|c|c|c|}
\hline ID & Description & p-value & FDR & q-value & Gene ID & Count \\
\hline hsa 04926 & Relaxin signaling pathway & 0.000164 & 0.008539 & 0.005791 & EGFR/MMP1/COL1A1/COL3A1 & 4 \\
\hline hsa 05219 & Bladder cancer & $9.60 \mathrm{E}-05$ & 0.008539 & 0.005791 & EGFR/CDKN1A/MMP1 & 3 \\
\hline hsa 05323 & Rheumatoid arthritis & 0.001086 & 0.024598 & 0.016681 & MMP3/MMP1/ICAM1 & 3 \\
\hline hsa 05215 & Prostate cancer & 0.001227 & 0.024598 & 0.016681 & MMP3/EGFR/CDKN1A & 3 \\
\hline \multirow[t]{2}{*}{ hsa 04933} & AGE-RAGE signaling pathway in & 0.00134 & 0.024598 & 0.016681 & ICAM1 /COL1A1/COL3A1 & 3 \\
\hline & diabetic complications & & & & & \\
\hline hsa 05146 & Amoebiasis & 0.001419 & 0.024598 & 0.016681 & HSPB1/COL1A1/COL3A1 & 3 \\
\hline
\end{tabular}

\section{Figures}




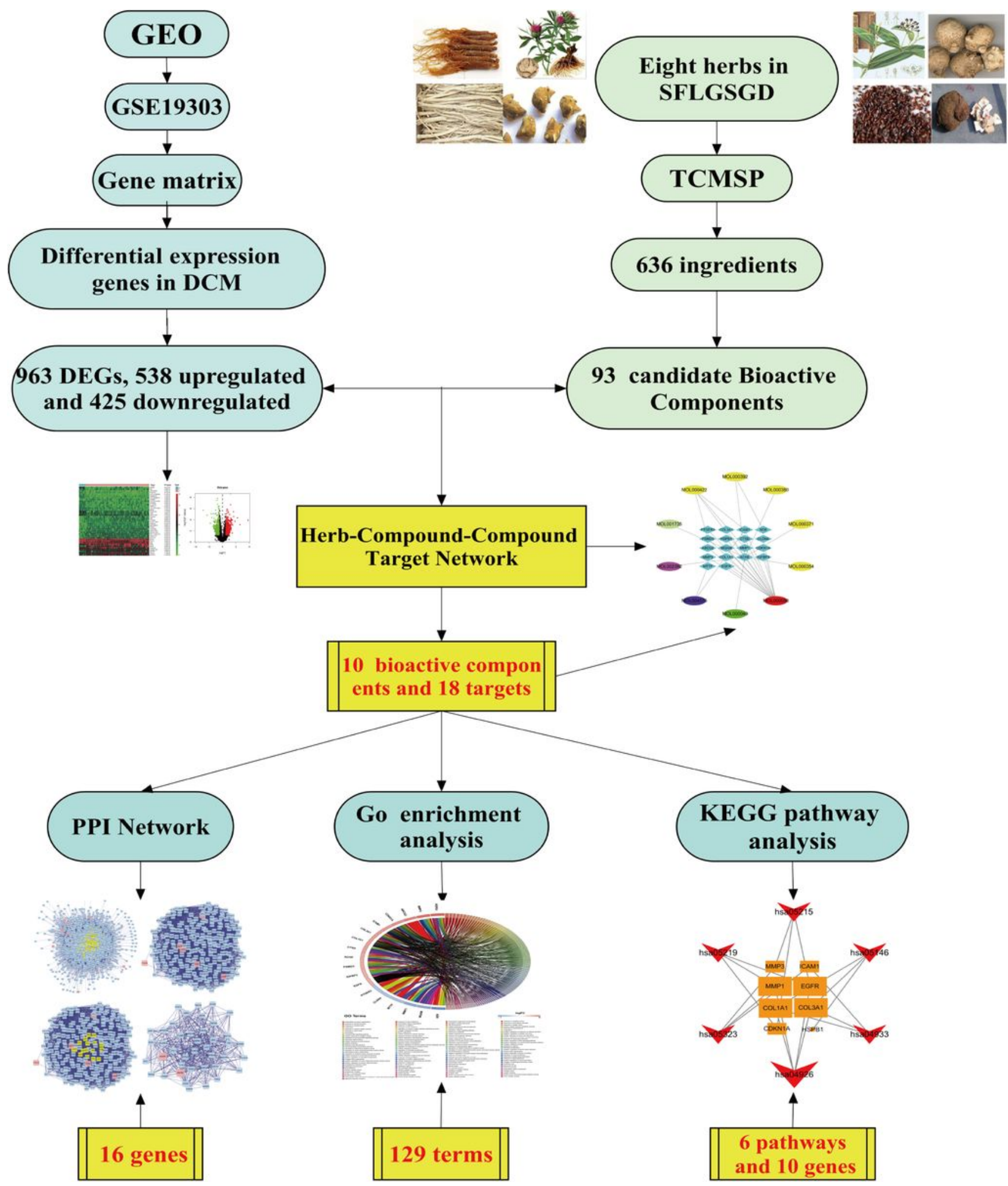

Figure 1

The flow chart of the current study. 


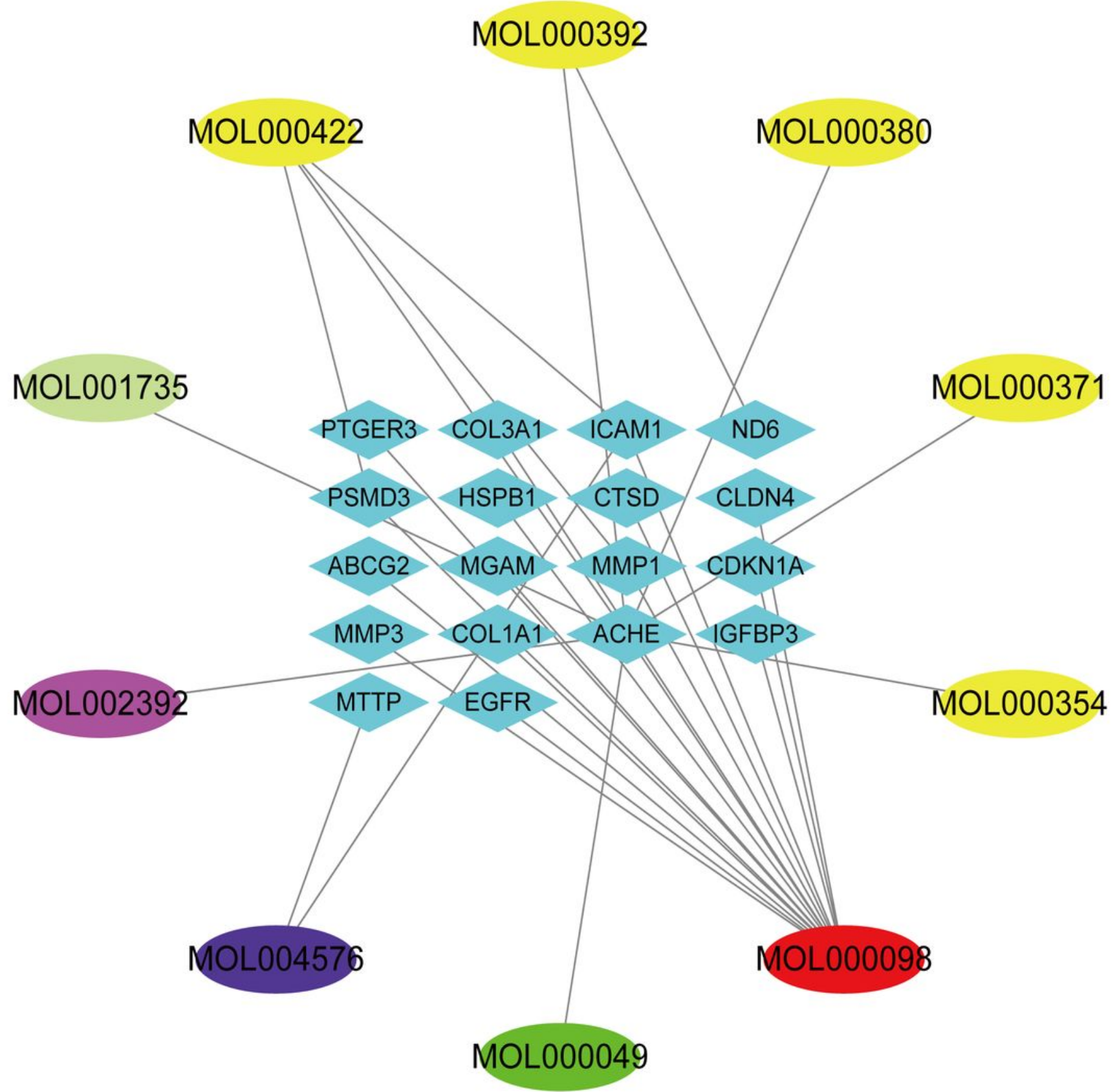

Figure 2

Regulatory network of TCM-Compound-Compound Targets. The ellipse nodes represent active compounds derived from herbs, Hedysarum Multijugum Maxim (yellow), Plantaginis Semen (pale green), Aconiti Lateralis Radix Praeparata (lilac), Cinnamomi Ramulus (purple), Atractylodes Macrocephala Koidz (green) and multiherbs (red). The blue diamond nodes represent targets. The edges represent the interactions between the active ingredients and targets. 

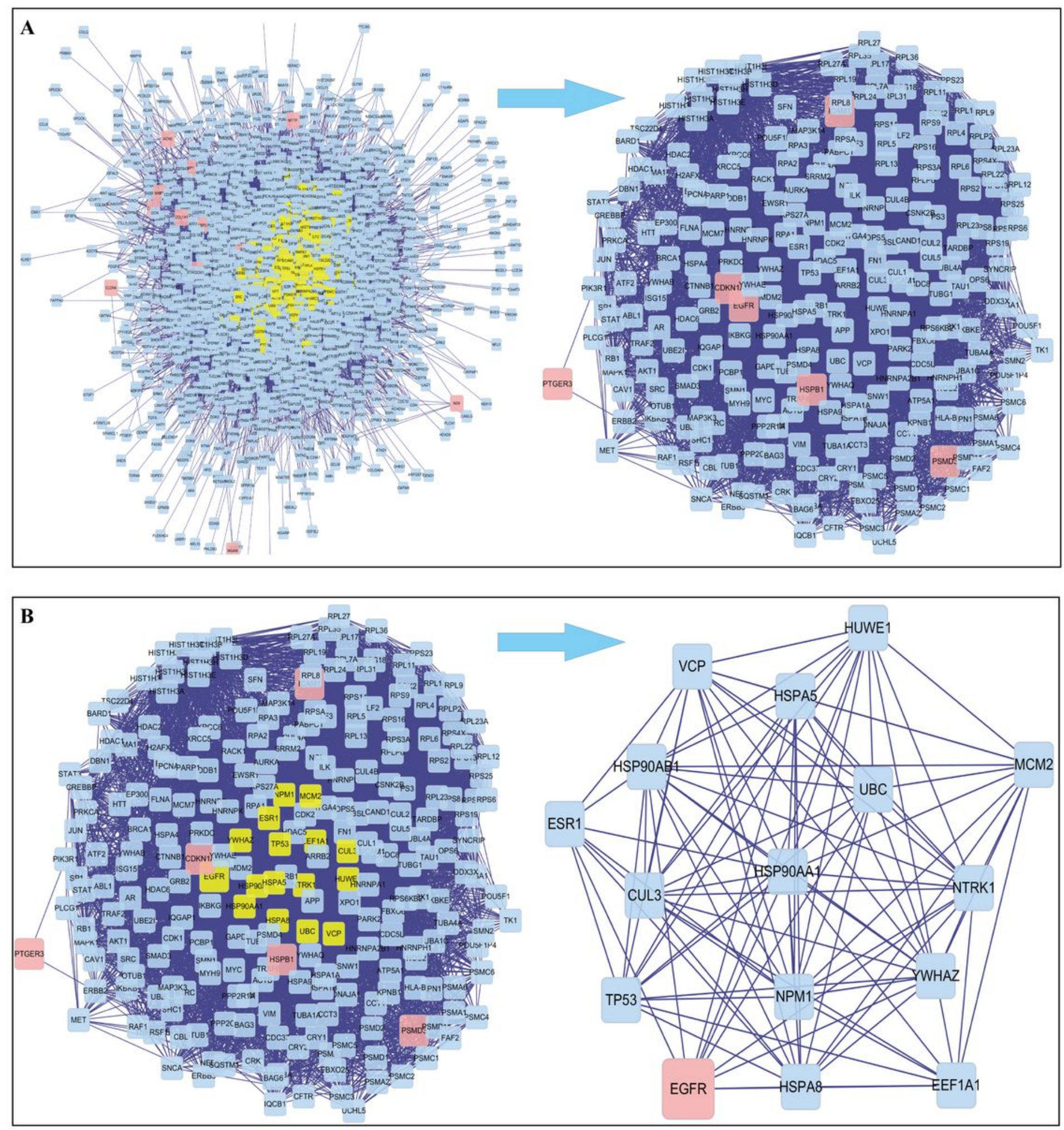

\section{Figure 3}

The procedure of topologically essential genes screened by CytoNCA. (A) The process of selected 249 genes ( $\mathrm{DC} \triangle 81)$. (B) The process of selected 16 genes $(\mathrm{BC} \triangle 600)$. The yellow nodes represent the selected genes, pink nodes represent the 18 targets, and blue nodes represent the neighbors. 


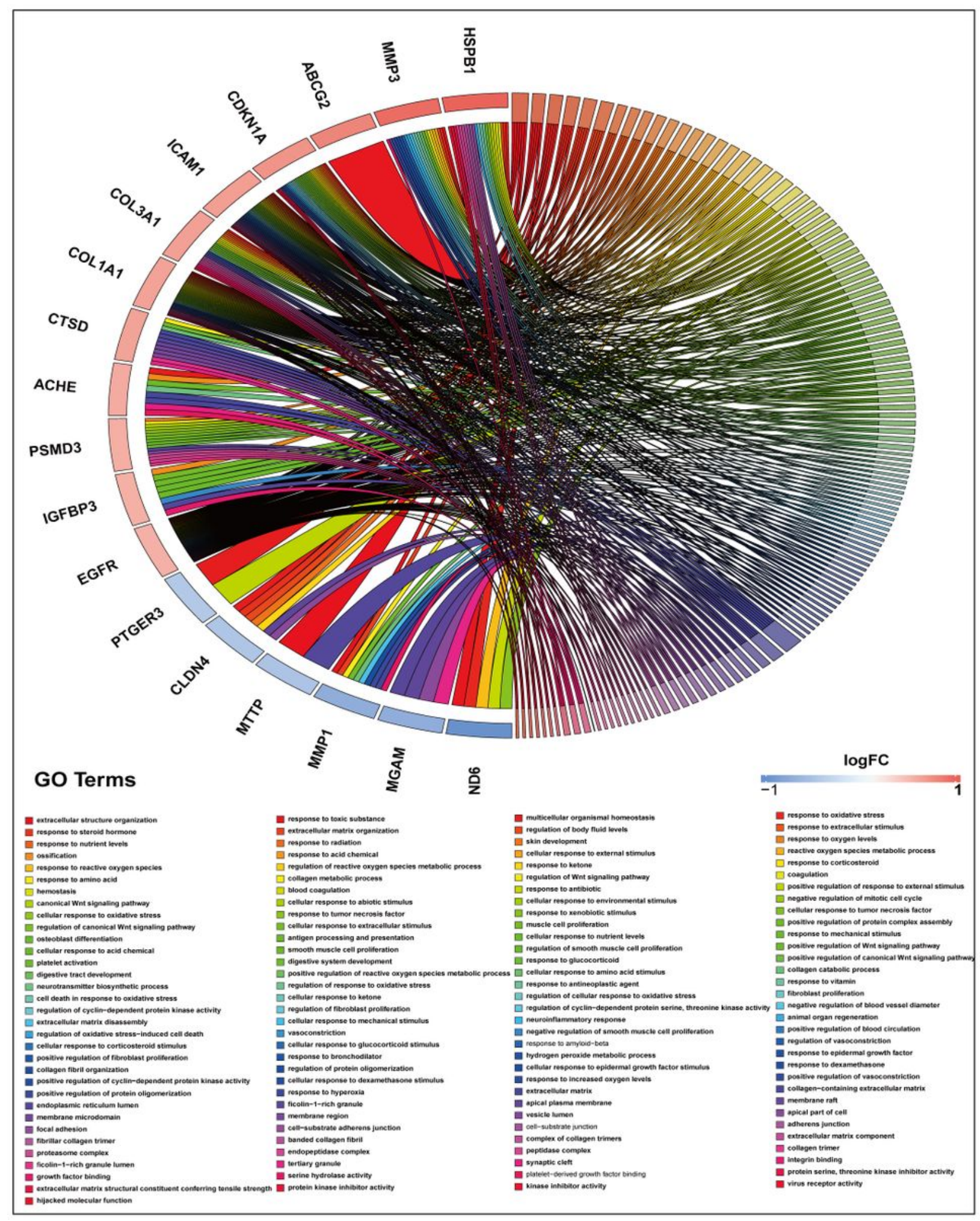

Figure 4

GO terms analysis of target genes. On the left outside of the circle are the genes, up-regulation was red, and down-regulation was blue, on the right was a different $\mathrm{GO}$ terms, and the genes linked via ribbons to their assigned terms. Small squares of various colors represent different terms (FDR<0.05). 


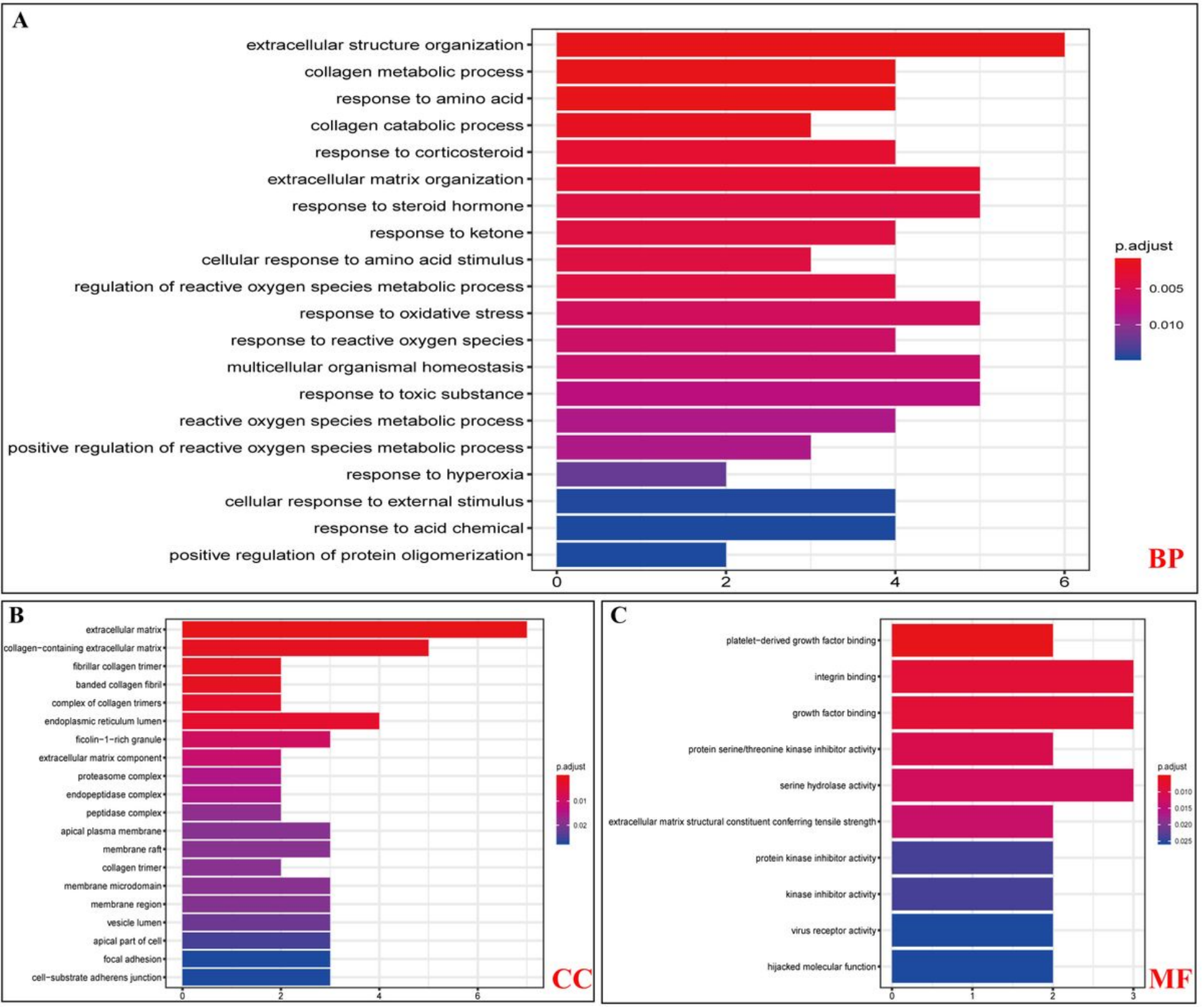

\section{Figure 5}

The 20 most significant gene ontology analysis of therapy target genes of SFLGZGD on DCM. (A) Biological Processes, (B) Cellular Components, (C) Molecular Functions. 

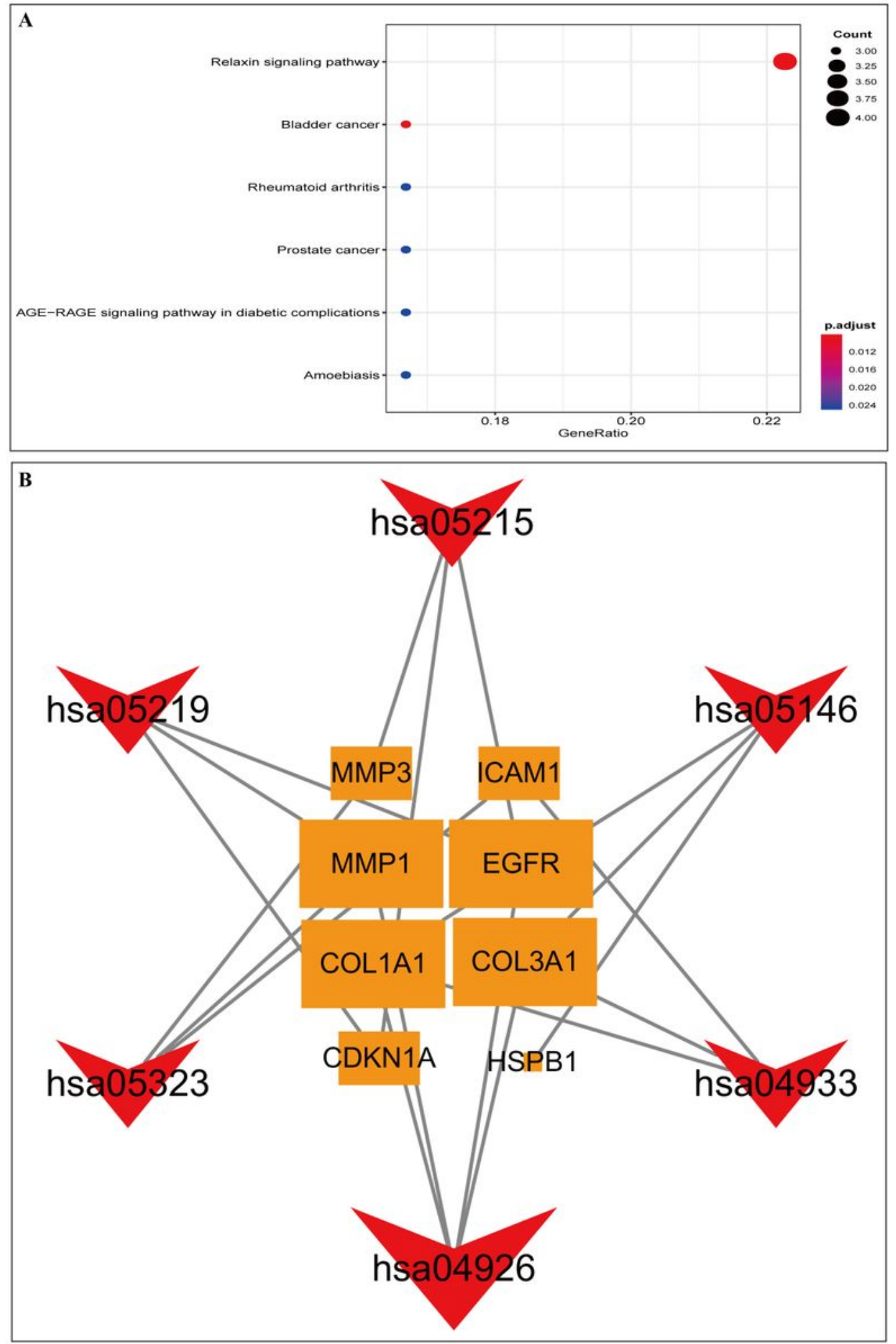

\section{Figure 6}

The KEGG pathway enrichment analysis and network of therapy target genes of SFLGZGD on DCM. (A) The KEGG pathway enrichment analysis. (B) The network between the target genes and pathways. The red inverted triangle nodes denote pathways, and the red nodes denote target genes. The edges represent the interactions between them, and node size is proportional to their degree. 